BMJ Open

Sport \&

Exercise

Medicine

\title{
Associations between fitness, physical activity and mental health in a community sample of young British adolescents: baseline data from the Fit to Study trial
}

Catherine Wheatley (D), ${ }^{1}$ Thomas Wassenaar (D, ${ }^{1}$ Piergiorgio Salvan, ${ }^{1}$ Nick Beale, ${ }^{2}$ Thomas Nichols, ${ }^{3}$ Helen Dawes, ${ }^{2}$ Heidi Johansen-Berg ${ }^{1}$

To cite: Wheatley C, Wassenaar $\mathrm{T}$, Salvan $\mathrm{P}$, et al. Associations between fitness, physical activity and mental health in a community sample of young British adolescents: baseline data from the Fit to Study trial. BMJ Open Sport \& Exercise Medicine 2020;0:e000819. doi:10.1136/bmjsem-2020000819

- Supplemental material is published online only. To view please visit the journal online (http://dx.doi.org/10.1136/ bmjsem-2020-000819).

Received 29 April 2020 Revised 21 July 2020 Accepted 13 September 2020

(C) Author(s) (or their employer(s)) 2020. Re-use permitted under CC BY. Published by BMJ.

For numbered affiliations see end of article.

Correspondence to Catherine Wheatley; catherine.wheatley@ndcn.ox.ac.uk

\section{ABSTRACT}

Objectives To examine relationships between fitness, physical activity and psychosocial problems among English secondary school pupils and to explore how components of physically active lifestyles are associated with mental health and well-being.

Methods A total of 7385 participants aged 11-13 took a fitness test and completed self-reported measures of physical activity, attitudes to activity, psychosocial problems and self-esteem during the Fit to Study trial. Multilevel regression, which modelled school-level cluster effects, estimated relationships between activity, fitness and psychosocial problems; canonical correlation analysis (CCA) explored modes of covariation between active lifestyle and mental health variables. Models were adjusted for covariates of sex, free school meal status, age, and time and location of assessments.

Results Higher fitness was linked with fewer internalising problems $(\beta=-0.23 ; 95 \% \mathrm{Cl}-0.26$ to $-0.21 ; p<0.001)$. More activity was also related to fewer internalising symptoms ( $\beta=-0.24 ; 95 \% \mathrm{Cl}-0.27$ to $-0.20 ; p<0.001$ ); the relationship between activity and internalising problems was significantly stronger for boys than for girls. Fitness and activity were also favourably related to externalising symptoms, with smaller effect sizes. One significant CCA mode, with a canonical correlation of $0.52(p=0.001)$, was characterised high cross-loadings for positive attitudes to activity (0.46) and habitual activity (0.42) among lifestyle variables; and for physical and global self-esteem ( 0.47 and 0.42) among mental health variables.

Conclusion Model-based and data-driven analysis methods indicate fitness as well as physical activity are linked to adolescent mental health. If effect direction is established, fitness monitoring could complement physical activity measurement when tracking public health.

\section{INTRODUCTION}

The youth inactivity crisis, and poor adolescent mental health, are pressing public health challenges. Only $22 \%$ of boys and $15 \%$ of girls achieve the daily hour of moderate-tovigorous physical activity (MVPA) recommended by the WHO, ${ }^{1}{ }^{2}$ and up to one in

\section{What are the new findings}

- Cardiorespiratory fitness, as well as physica activity, is linked to adolescent mental health.

- Fitness is more strongly associated with internalising than externalising symptoms of psychological distress.

- Physically active lifestyles incorporating positive attitudes to activity are linked to better mental health, characterised by higher self-esteem and fewer internalising problems.

How might findings impact practice in the future

- Paediatric public health surveillance could include measures of fitness as well as physical activity.

- Interventions targeting adolescent fitness could be tested for effects on both mental and physical health.

five young people will experience mental illhealth $^{3}$ : half of all disorders are established by age $14 .^{4}$

Regular physical activity has a positive impact on mental health, but the relationship is complex and not fully understood..$^{5}$ Poor mental health has numerous causes including biological, psychological and social-environmental factors, such as social and economic deprivation ${ }^{6}$ : the benefits of exercise for mental health may depend on how individuals experience activity, and the context in which it takes place. ${ }^{7}$ In adolescent clinical samples, aerobic exercise programmes can treat depression and improve self-esteem, which can protect against the onset of disorders. ${ }^{89}$ In the general adolescent population, evidence of links between physical activity and mental health, while promising, is inconclusive. ${ }^{10}$ Studies involving large community samples have found variously that vigorous activity is unrelated to anxiety and depression 
but linked with better social functioning ${ }^{11}$; MVPA is associated with lower (but not clinically meaningful) levels of depression and anxiety, and greater well-being ${ }^{12}$; MVPA is linked to fewer emotional and peer-related problems in primary school children but more conduct and hyperactivity-inattention difficulties ${ }^{13}$; and meeting daily MVPA guidelines is related to fewer global psychosocial problems at clinically meaningful levels. ${ }^{14}$ Girls and boys appear to respond differently: more peer and emotional problems have been linked with lower activity among boys, but not girls. $^{13}$

Cardiorespiratory fitness (CRF) is a modifiable risk factor linked to current and future physical health among adolescents, independent of physical activity. ${ }^{15-17}$ Much less is known about the role of CRF in adolescent mental health, ${ }^{18}$ although it has been linked to a reduced incidence of depression in young people, potentially via better brain health. ${ }^{15}$ Research linking fitness and psychosocial problems in community samples are sparse: two very recent studies found small but significant negative associations between fitness and psychosocial problems: the relationship was stronger among girls than boys. ${ }^{20} 21$

Evidence linking fitness and mental health would support calls to extend paediatric public health surveillance to include measures of both fitness and physical activity. ${ }^{22} 23$ Fitness tests linked to peak oxygen consumption provide objective, clearly defined measures capturing long-term active lifestyles, whereas accelerometry or self-report measures of physical activity provide 7-day snapshots influenced by circumstances, activity type and recall bias. ${ }^{24}$ Fitness also has potential to capture the interplay of other factors linked to mental health, including obesity. ${ }^{25}$

Understanding more about relationships between activity, fitness and mental health in young adolescents is therefore important. Our objectives in this study were to examine associations between psychosocial problems and physical activity, and, separately, psychosocial problems and CRF in a community sample of adolescents. Because evidence suggests these relationships might differ between girls and boys, and because deprivation can impact mental health, we also wished to explore whether associations vary with sex and socioeconomic status. Given the complex relationship between activity, fitness, contextual factors and mental health, we also used a data-driven approach to explore whether physically active lifestyles, including positive attitudes towards regular physical activity leading to higher fitness, are associated with good mental health, characterised by few psychosocial problems and positive self-esteem.

\section{METHOD}

Design

The study was a cross-sectional analysis of baseline data from the Fit to Study cluster-randomised controlled trial, registered with ClinicalTrials.gov, NCT03286725. Trial recruitment, methodology and consent procedures are reported elsewhere ${ }^{26}$ in line with the Consolidated Standards for Reporting Trials (CONSORT).

\section{Participants}

Baseline assessments of pupils aged 11-13 from British state secondary schools took place between June and September 2017, at the end of Year 7 and the start of Year 8. Schools provided participants' sex, birth date and their eligibility for free school meals (eFSM), an indicator of socioeconomic disadvantage. The full sample recruited at baseline was $\mathrm{n}=16$ 017; male=7056; eFSM=3068 .

\section{Outcome measures}

Cardiorespiratory fitness was assessed during school physical education (PE) lessons with the $20 \mathrm{~m}$ Multistage Fitness Test ${ }^{27}$ validated in numerous studies. ${ }^{28}$ Scores were number of laps completed: pupils scoring at or above normative 50th percentile scores for boys and girls aged 12 were considered fit. ${ }^{29}$

Physical activity (past week) was measured with a validated, single-item measure. ${ }^{30}$ Participants reporting at least $60 \mathrm{~min}$ ' MVPA for each of the past 7 days were considered active; all others were inactive. Habitual physical activity (past 6 months) was measured with a single self-report item. ${ }^{31}$ In line with established methods, ${ }^{32}$ attitude to physical activity was the mean score of three items on a visual analogue scale (boring-fun; stressful-relaxing; dissatisfying-satisfying). Higher scores indicate better outcomes.

Psychosocial problems were measured with the Strengths and Difficulties Questionnaire (SDQ) ${ }^{33}$ valid and reliable in children aged over $11^{34}$ consisting of 25 items measuring five subscales: conduct problems, hyperactivity-inattention, peer-relationship problems, emotional symptoms and pro-social behaviour. We used combined conduct and hyperactivity scores (externalising) and peer and emotional scores (internalising) as primary outcome measures for model-based analyses because there is evidence that in low-risk samples, the more focused subscales might not tap into distinct aspects of mental health. ${ }^{35}$ We interpreted internalising scores $\geq 9$ and externalising scores $\geq 11$ as high/very high. ${ }^{35}$

Self-esteem was measured with the global and physical self-esteem subscales of the short Physical Self Description Questionnaire, validated for use among adolescents and typically used in PE contexts; higher scores indicate better outcomes. ${ }^{36}$ Cronbach's alpha is reported for scales and subscales in table 1.

\begin{tabular}{lll}
\hline Table 1 & Cronbach's alphas for & psychosocial variables \\
\hline Scale & Number of items & $\boldsymbol{\alpha}$ \\
\hline SDQ-Internalising score & 10 & 0.75 \\
SDQ-Externalising score & 10 & 0.80 \\
SDQ-Emotional & 5 & 0.72 \\
SDQ-Peer & 5 & 0.62 \\
SDQ-Conduct & 5 & 0.64 \\
SDQ-Hyperactivity-inattention & 5 & 0.74 \\
SDQ-Pro-social & 5 & 0.64 \\
Global self-esteem & 5 & 0.81 \\
Physical self-esteem & 3 & 0.91 \\
Attitude to activity & 3 & 0.81 \\
\hline
\end{tabular}




\section{Statistical analyses}

Primary analyses included participants who completed all outcome measures. For secondary analyses, we used the full sample available at baseline imputing missing data to account for potential bias. Secondary analyses based on multiple imputations are reported in online supplemental material.

\section{Categorical analyses by sex and eFSM}

The odds of scoring in the high/very high or typical category for internalising and externalising problems and the odds of being active ( 7 days) or fit ( $\geq 50$ th percentile score) by sex and eFSM status were calculated using multilevel logistic regression, controlling for school clusters.

\section{Model-based analyses}

We used multilevel linear regression, with random intercepts to account for school clusters, to explore associations between fitness, physical activity and internalising and externalising problems. Because assessments took place in both PE and form classes, we did not include random intercepts for class clusters. Data were z-scored to facilitate comparisons within and between models. The distribution of internalising and externalising scores was positively skewed: we applied a square-root transformation and a rank inverse normal transformation, respectively, to these data. We developed four separate models, with internalising and then externalising scores as outcome variables, and physical activity and then fitness as predictor variables. Then, using forced entry, we fitted four adjusted models, including fixed effects for sex, eFSM and their interaction terms, and age and nuisance covariates, which were term of fitness assessment, term of questionnaire assessment and location of questionnaire assessment. Where interactions were non-significant, we removed these terms and re-ran the model. Where significant interactions with sex and/or eFSM were present $(\mathrm{p}<0.0125$; Bonferroni-corrected for four comparisons), we explored these by plotting their slopes. We obtained crude parameter estimates by developing unadjusted models with data that was transformed but not standardised. Analyses were conducted in $\mathrm{R}$ with the lme4 package. $^{37}$

\section{Reverse transformations}

To derive an approximate value for change in internalising and externalising scores associated with a one-lap increase in fitness score and an additional day per week of activity, we multiplied the transformed estimates in each model by the $\mathrm{SD}$ of the distribution on the original scale.

\section{Data-driven analysis of associations}

We used canonical correlation analysis (CCA) -an approach which measures associations between two sets of variables, making no prior assumptions about relationships-to explore modes of covariation between physically active lifestyles and mental health. The active lifestyle variables were measures of activity, fitness, habitual activity and attitude to activity. The mental health variables were the conduct, hyperactivity, peer, emotional and pro-social sub-scales from the SDQ; global self-esteem and physical self-esteem. The CCA maximised the strength of correlation $\left(R_{c}\right)$ between a linear combination of active lifestyle measures and a linear combination of mental health metrics. For every covariation mode, we characterised the canonical cross-loading or the degree of influence that each variable exerted on its opposite canonical variate $\left(R_{s}\right)$. Better mental health would be indicated by negative $R_{s}$ values for psychosocial problems and positive values for self-esteem. We took the mean squared error (MSE) as an estimate of the accuracy of the match between predicted and actual data sets. For significant CCA modes, we calculated the redundancy index or the amount of variance in one group of variables explained by the other group. Significance of CCA modes was assessed through permutations ${ }^{38}$; analyses were conducted using Matlab canoncor.

\section{Patient and public involvement}

Teachers advised on the feasibility of data collection, administered fitness tests and supervised questionnaire completion. Schools received trial progress reports. Teachers were invited to attend academic conferences.

\section{RESULTS}

After data cleaning, 7385 participants (female $=4441$; $60.1 \%$; eFSM=1044; 14.1\%) from 67 schools completed the questionnaire and fitness test (online supplemental material figure 1); $95.5 \%$ answered the questionnaire and $85.4 \%$ took the fitness test before the summer vacation; $93.8 \%$ completed the survey in school. Mean age at the start of the school year was 12.5 (SD 0.29) years. Mean scores on each variable and percentages in each category stratified by sex and eFSM status are presented in table 2 .

High internalising scores were significantly associated with sex but not eFSM: odds of a high score were 1.6 times greater for girls (table 3 ). The odds of high externalising scores were lower for girls and higher for disadvantaged pupils. The odds of being active daily were higher for boys but not for disadvantaged pupils. The odds of meeting or exceeding normative fitness levels were lower for eFSM pupils, but there was no significant difference between sexes.

Models 1 and 2 examined internalising symptoms and their relationship with physical activity and fitness, respectively (table 4). There was a significant effect of sex on the relationship between internalising symptoms and activity: the favourable relationship was stronger for boys than girls $(\beta=0.07 ; 95 \%$ CI 0.03 to $0.12 ; p<0.001)$. More activity was significantly associated with fewer internalising symptoms $(\beta=-0.24 ; 95 \% \mathrm{CI}-0.27$ to $-0.20 ; \mathrm{p}<0.001)$. Figure 1 plots the interaction of physical activity and sex: for every unit increase in activity, boys showed a significantly larger reduction in internalising symptoms.

Higher fitness was significantly related to fewer internalising symptoms $(\beta=-0.23 ; 95 \%$ CI -0.26 to -0.21 ; 
Table 2 Mean (SD) participant scores on all outcome variables ( $n=7385)$

\begin{tabular}{|c|c|c|c|c|c|c|}
\hline Variable & Range & Male(n=2944) & Female $(n=4441)$ & No $\operatorname{FSM}(n=6341)$ & eFSM(n=1044) & Overall $(n=7385)$ \\
\hline Internalising score & $0-20$ & & & & & \\
\hline Mean (SD) & & $4.5(3.3)$ & $5.4(3.5)$ & $5.0(3.4)$ & $5.4(3.7)$ & $5.1(3.4)$ \\
\hline High/V High (\%) & & 13.1 & 19.0 & 16.3 & 19.1 & 16.7 \\
\hline Externalising score & $0-20$ & & & & & \\
\hline Mean (SD) & & $6.6(3.8)$ & $5.9(3.6)$ & $6.1(3.7)$ & $7.0(3.8)$ & $6.2(3.7)$ \\
\hline High/V High (\%) & & 17.0 & 12.5 & 13.4 & 19.8 & 14.3 \\
\hline Conduct problems & $0-10$ & & & & & \\
\hline Mean (SD) & & $2.3(1.9)$ & $1.9(1.7)$ & $2.0(1.8)$ & $2.5(1.9)$ & $2.1(1.8)$ \\
\hline Hyperactivity & $0-10$ & & & & & \\
\hline Mean (SD) & & $4.3(2.4)$ & $4.0(2.3)$ & $4.1(2.3)$ & $4.5(2.4)$ & $4.1(2.4)$ \\
\hline Peer problems & $0-10$ & & & & & \\
\hline Mean (SD) & & $2.0(1.8)$ & $1.8(1.7)$ & $1.9(3.1)$ & $2.1(1.9)$ & $1.9(1.8)$ \\
\hline Emotional symptoms & $0-10$ & & & & & \\
\hline Mean (SD) & & $2.6(2.1)$ & $3.7(2.4)$ & $3.2(2.3)$ & $3.4(2.4)$ & $3.2(2.3)$ \\
\hline Prosocial behaviour & $0-10$ & & & & & \\
\hline Mean (SD) & & $7.3(1.8)$ & $8.0(1.7)$ & 7.7(1.8) & $7.4(1.8)$ & $7.7(1.8)$ \\
\hline Fitness & $1-137$ & & & & & \\
\hline Mean (SD) & & $42.8(22.7)$ & $32.5(16.1)$ & $37.5(19.9)$ & $30.5(17.1)$ & $36.5(19.6)$ \\
\hline$\% \geq$ normative & & 49.0 & 53.8 & 55.6 & 39.2 & 51.9 \\
\hline Physical activity & $0-7$ & & & & & \\
\hline Mean (SD) days & & $4.7(1.9)$ & 4.3(1.9) & $4.5(1.9)$ & $4.2(2.0)$ & $4.4(1.9)$ \\
\hline$\%$ active daily & & 24.5 & 15.9 & 19.4 & 18.3 & 19.3 \\
\hline Habitual activity & $1-7$ & & & & & \\
\hline Mean (SD) & & $5.3(1.4)$ & $5.1(1.4)$ & $5.2(1.4)$ & $4.9(1.5)$ & $5.2(1.4)$ \\
\hline Attitude to activity & $1-7$ & & & & & \\
\hline Mean (SD) & & $5.2(1.2)$ & $4.9(1.2)$ & $5.0(1.2)$ & $4.8(1.3)$ & $5.0(1.2)$ \\
\hline Global self esteem & $1-6$ & & & & & \\
\hline Mean (SD) & & $4.5(0.87)$ & $4.4(0.92)$ & $4.4(0.89)$ & $4.3(0.96)$ & $4.4(0.90)$ \\
\hline Physical self esteem & $1-6$ & & & & & \\
\hline Mean (SD) & & 4.5(1.2) & 4.3(1.3) & 4.4(1.3) & 4.3(1.3) & $4.4(1.3)$ \\
\hline
\end{tabular}

Table 3 ORs of psychosocial problems, activity and fitness by sex and disadvantage

\begin{tabular}{lll}
\hline & Sex* OR $^{(C I)}$ & eFSM† OR (CI) \\
\hline $\begin{array}{l}\text { High internalising } \\
\text { score }\end{array}$ & $1.60^{\star * *}(1.39-1.84)$ & $1.17(0.98-1.39)$ \\
$\begin{array}{l}\text { High externalising } \\
\text { score }\end{array}$ & $0.73^{\star * *}(0.63-0.84)$ & $1.56^{\star * *}(1.31-1.86)$ \\
Active & & \\
Fit & $0.58^{\star * *}(0.51-0.65)$ & $0.95(0.80-1.13)$ \\
\hline
\end{tabular}

${ }^{*}$ Reference category: male.

†Reference category: not eligible for FSM.

${ }^{* * *} \mathrm{p}<0.0001$.

$\mathrm{p}<0.001)$. There were no significant interactions between fitness and either sex or eFSM at the $\mathrm{p}=0.0125$ threshold. School effects explained $2.8 \%$ of variance in internalising symptoms in both models. Model 1 explained $6.2 \%$ of variance in internalising symptoms over and above school effects, while Model 2 explained $7.3 \%$.

Models 3 and 4 (table 3) examined externalising symptoms and their relationship with physical activity and fitness, respectively. More activity was significantly related to fewer externalising symptoms ( $\beta=-0.09 ; 95 \%$ CI -0.11 to -0.06 ; $\mathrm{p}<0.001)$. There were no significant interactions between sex, eFSM and activity. Higher fitness is significantly associated with fewer externalising symptoms $(\beta=-0.07 ; 95 \% \mathrm{CI}$ -0.09 to $-0.04 ; \mathrm{p}=0.002)$. There was no significant interaction of sex or eFSM at the $\mathrm{p}=0.0125$ threshold.

School effects explained $3.0 \%$ of variance in externalising symptoms. Model 3 explained $3.1 \%$ of variance in externalising symptoms over and above school effects, while Model 4 explained 2.9\%.

Reverse-transforming estimates for physical activity and fitness showed an extra day per week of physical activity is linked to internalising and externalising scores that are lower by 0.85 and 0.20 , respectively. An additional lap on the fitness test is associated with 
Table 4 Estimates of associations between physical activity, fitness, internalising and externalising symptoms ( $\mathrm{n}=7385$ )

\begin{tabular}{|c|c|c|c|c|c|c|c|}
\hline \multicolumn{4}{|l|}{ Adjusted† } & \multicolumn{4}{|l|}{ Unadjusted } \\
\hline Predictor variables & $\boldsymbol{\beta}$ & $2.5 \% \mathrm{Cl}$ & $97.5 \% \mathrm{Cl}$ & Predictor variables & $\beta$ & $2.5 \% \mathrm{Cl}$ & $97.5 \% \mathrm{Cl}$ \\
\hline \multicolumn{8}{|l|}{ Model 1} \\
\hline eFSM§ & $0.07^{*}$ & -0.00 & 0.13 & eFSM§ & 0.07 & -0.01 & 0.13 \\
\hline Sexף & $0.24^{\star \star \star}$ & 0.19 & 0.28 & Sexף & 0.06 & -0.05 & 0.18 \\
\hline Fit & $-0.23^{\star \star \star}$ & -0.26 & -0.21 & Fit & $-0.01^{\star \star \star}$ & -0.01 & -0.01 \\
\hline eFSM§ & 0.03 & -0.04 & 0.09 & eFSM§ & 0.03 & -0.03 & 0.09 \\
\hline $\operatorname{Sex} \|$ & $0.16^{\star \star \star}$ & 0.11 & 0.21 & Sexף & $0.15^{\star \star \star}$ & 0.10 & 0.20 \\
\hline \multicolumn{8}{|l|}{ Model 3} \\
\hline PA & $-0.09^{\star \star \star}$ & -0.11 & -0.06 & PA & $-0.05^{\star \star \star}$ & -0.06 & -0.03 \\
\hline Fit & $-0.07^{\star \star}$ & -0.09 & -0.04 & Fit & $-0.003^{\star \star \star}$ & -0.004 & 0.001 \\
\hline eFSM§ & $0.21^{\star \star}$ & 0.15 & 0.28 & eFSM§ & $0.22^{\star \star \star}$ & 0.15 & 0.28 \\
\hline Sexף & $-0.21^{\star \star \star}$ & -0.26 & -0.15 & Sexף & $-0.21^{\star \star \star}$ & -0.26 & -0.16 \\
\hline
\end{tabular}

†Fully adjusted multilevel model including age, sex, eFSM, nuisance covariates and school effects; physical activity/fitness, internalising and externalising scores are z-scored; internalising symptoms are square-root transformed and externalising scores are rank-inverse transformed to account for positively skewed skewed distribution.

¥Unadjusted multilevel models including transformed but unscaled data for PA, fitness, sex and eFSM; controlled for school effects.

§Reference category: not eligible for FSM.

१Reference category: male.

${ }^{* * *} \mathrm{p}<0.001 ;{ }^{* *} \mathrm{p}<0.0125$.

eFSM, eligible for free school meals; Fit, cardiovascular fitness; PA, physical activity.

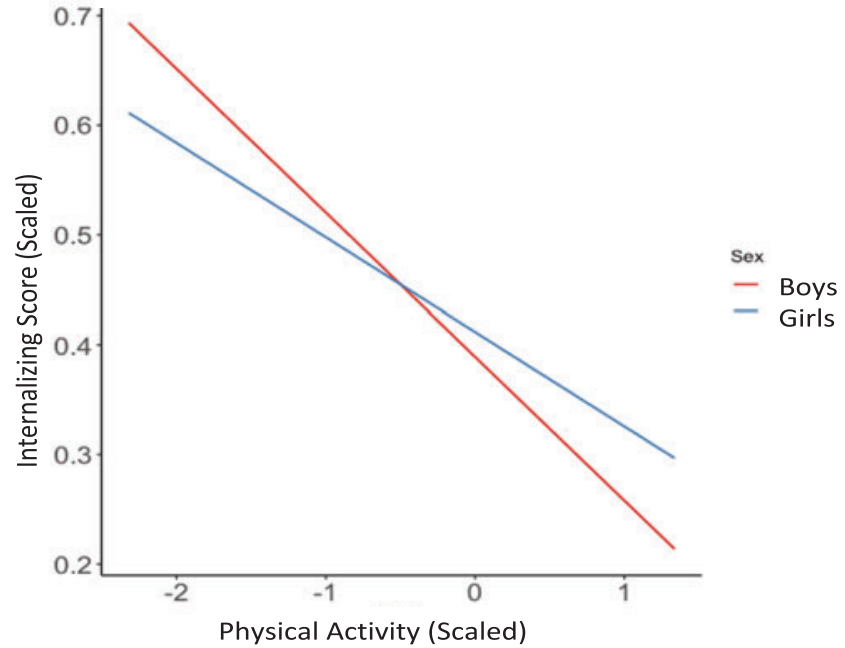

Figure 1 Fully adjusted multilevel model including age, sex, eFSM, nuisance covariates and school effects; physical activity and internalising scores are z-scored; internalising symptoms are square-root transformed toaccount for positively skewed distribution.

internalising and externalising scores that are lower by 0.77 and 0.18 .

In the full-imputed dataset, fitness and physical activity were significantly related to both internalising and externalising symptoms $(\mathrm{p}<0.001)$ but estimates were larger for fitness than for activity (online supplemental file). In the externalising models, estimates for fitness and physical activity were approximately twice the magnitude of those based on complete cases only. School effects explained similar variance in internalising and externalising symptoms.

CCA identified two statistically significant and meaningful modes of covariation between physically active lifestyle and mental health factors. The first mode, $\left(R_{c}\right.$ $=0.52, M S E=0.99, \mathrm{p}=0.001)$, was characterised by high cross-loadings for positive attitudes to MVPA $\left(R_{s}\right.$ $=0.46)$ and habitual activity $\left(R_{s}=0.42\right)$ in the activelifestyle variate, and physical self-esteem $\left(R_{s}=0.47\right)$ and global self-esteem $\left(R_{s}=0.42\right)$ in the mental health variate. Figure 2A presents variables' cross-loadings (online sup plemental file gives associated numerical data). In this mode, both physical activity $\left(R_{s}=0.35\right)$ and fitness $\left(R_{s}\right.$ $=0.28$ ) were influential. Low levels of internalising difficulties (emotional $R_{s}=-0.28$ and peer $R_{s}=-0.26$ ) had higher cross-loadings than low externalising difficulties (conduct $R_{s}=-0.15$ and hyperactivity $R_{s}=-0.12$ ). The scatter plot shows a canonical correlation between lifestyle and mental health for both boys (blue) and girls (red).

In the second mode $\left(R_{c}=0.34, M S E=2.60, \mathrm{p}=0.001\right)$, described in figure 2B (and online supplemental file), sex had high cross-loadings in the lifestyle variables 
(A)
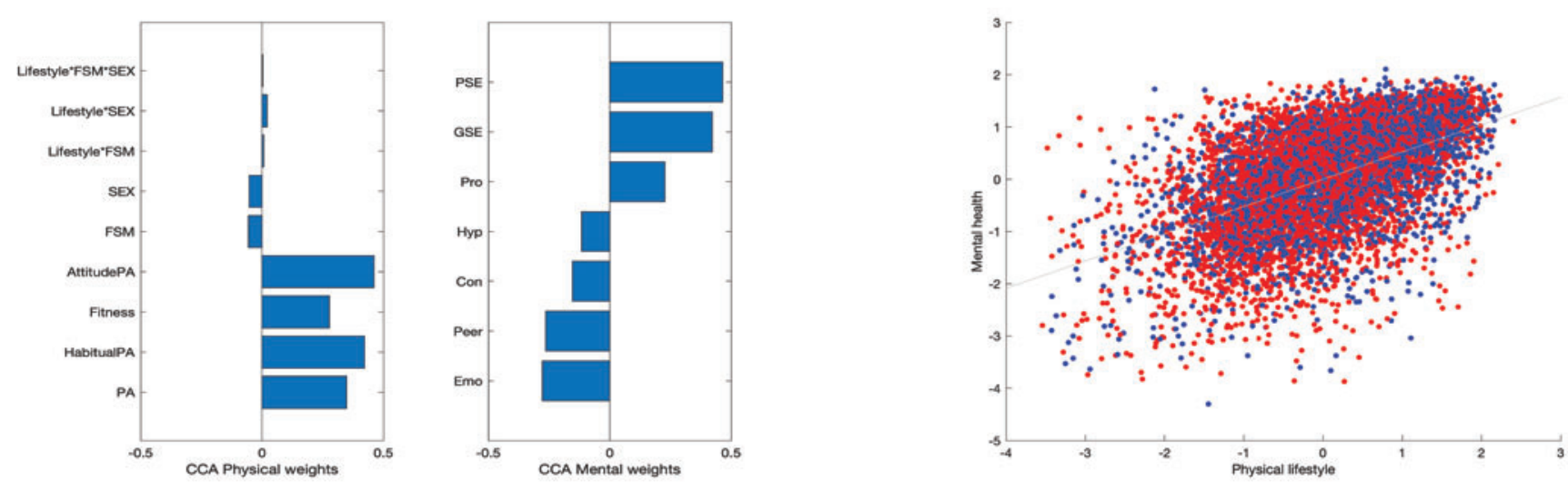

(B)
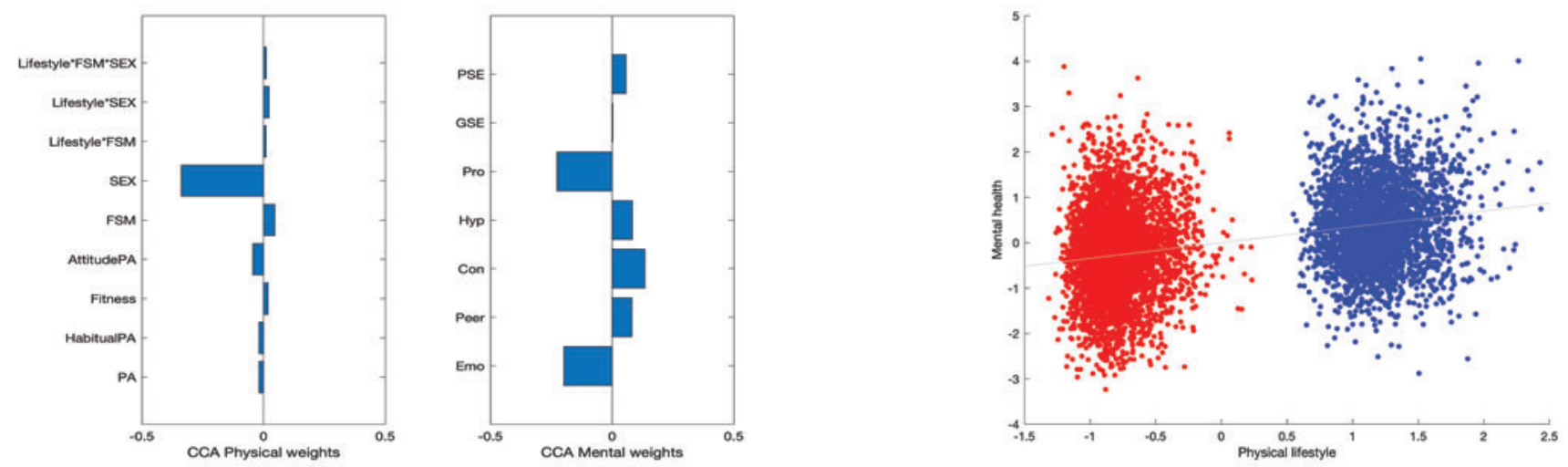

SEX reference category: male

FSM reference category: not eligible for FSM

Lifestyle*FSM $=$ the interaction of mean lifestyle variables and eFSM

Lifestyle*SEX $=$ the interaction of mean lifestyle variables and sex

Lifestyle*FSM*SEX = the interaction of mean lifestyle variables, eFSM and sex

Figure 2 (A) Canonical cross-loading of variables onto their respective variates in mode 1. (B) Canonical cross-loading of variables onto their respective variates in mode 2 .

$\left(R_{s}=-0.34\right)$ while pro-social behaviour $\left(R_{s}=-0.23\right)$ and emotional difficulties $\left(R_{s}=-0.20\right)$ were prominent across mental health variables. The scatterplot showed a clear difference between girls (red) and boys (blue): girls have a different pattern of mental health to boys, with higher predicted scores for both pro-social behaviour and emotional difficulties.

The redundancy index, or the amount of explained variance, was $8 \%$ for active lifestyle variables given mental health variables and $11 \%$ for the mental health set given the active lifestyle set.

\section{DISCUSSION}

We believe this is the first study to show a significant relationship between CRF and mental health in a community sample of young British adolescents. Higher fitness, and more days per week of activity, are both associated with fewer psychosocial problems in this group. The relationship between activity and internalising symptoms was stronger for boys than for girls, but none of the other relationships we explored varied significantly with either sex or socioeconomic status. A physically active lifestyle is linked to better mental health: positive attitudes towards activity, and habitual activity, are important features of the relationship, together with positive selfesteem. These are important findings because they suggest that fitness assessments could complement the physical activity measures that are essential for public health monitoring.

\section{Comparisons with other studies}

Our results add to the sparse research linking fitness and psychosocial problems in the adolescent population. We found a significant relationship with both internalising and externalising problems after controlling for sex, although the effect size was larger for internalising symptoms. This aligns with previous research: a recent Canadian study found a small favourable association between CRF and internalising problems, but only among girls, ${ }^{20}$ while an Australian study of older adolescents found the 
link between internalising symptoms and fitness was significantly stronger among girls. $^{21}$ Neither found a relationship with externalising symptoms.

Our findings that physical activity is favourably related to internalising and externalising symptoms supports previous research and strengthens the evidence of a favourable link between activity and mental health in the adolescent population.

Nevertheless, the range of measures and confounding variables under consideration make effect size comparisons difficult. In our study, the relationship between activity and internalising symptoms was stronger for boys, which aligns with previous findings that more peer and emotional problems have been linked with lower activity among boys, but not girls. ${ }^{13}$

We believe this is the first data-driven CCA to show that physically active lifestyles are linked to good mental health. Activity-positive attitudes and habitual activity were important features of the relationship, together with positive self-esteem, which can protect against onset of disorders. ${ }^{39}$ Our findings are supported by previous research highlighting favourable links between activity and physical self-esteem. ${ }^{40}$ A second mode of covariation revealed an association between sex and mental health: being female was linked to more pro-social behaviour but also more emotional difficulties, in line with UK norms. ${ }^{41}$

\section{Possible explanations and implications}

Physical activity, fitness and mental health are thought to be linked through several distinct mechanisms. CRF may promote stress resilience by optimising neuroendocrine and physiological responses to physical and also psychosocial stressors. ${ }^{42}$ Fitness may also contribute to improved mood via enhanced brain plasticity and growth factor expression. ${ }^{43}$ Separately, physical activity is thought to enhance mood and protect against depression. But these relationships appear to be bi-directional: cohort studies show that higher internalising scores, especially among boys, predict lower activity levels during adolescence. ${ }^{13}$

While our findings cannot prove causal relationships, our research indicates that interventions to boost fitness as well as activity, and promote positive attitudes to such behaviour, might have potential to reduce emotional and peer-related problems in particular among early adolescents from all socio-economic backgrounds, and especially boys. This is important, given that half of all disorders appear by age $14 .^{4}$

The variance in psychosocial problems explained by our models (which include sex and eFSM status, and control for school effects, age and nuisance variables) is less than $10 \%$ in all cases. But unlike other hypothesised causes of poor mental health in this age group, such as genetic predisposition or social-environmental circumstances, low fitness and activity are particularly good targets for interventions in community settings, including schools. ${ }^{44}$ Previous studies have attempted to establish whether membership of a broad physical activity category—being 'inactive' or 'active daily' for example—has a clinically meaningful impact on psychosocial problems, sometimes defined as a one-point change in the Total Difficulties Score, which is the sum of internalising and externalising scores. ${ }^{12-14}$ We found that an extra day of activity and an extra lap on the fitness test is linked with internalising scores that are lower by approximately 0.8 and externalising scores by around 0.2. Such a difference might be critical for individuals at high risk of a clinical disorder, at the margin between slightly raised and high scores. Furthermore, there is growing support for a population approach to adolescent mental health that promotes well-being and healthy behaviours among all young people ${ }^{45}$ : such an approach would aim to shift the entire distribution rather than targeting individuals at key cut-off points on the curve.

One potential explanation for the closer link that activity and fitness have with internalising symptoms is that regular activity offers an outlet for adolescents with hyperactivity-inattention and conduct problems: these challenging behaviours are sometimes associated with higher movement rather than the inverse. ${ }^{46}$ The relationship between female sex and mental health could explain why the favourable relationship between activity and internalising symptoms appears stronger for boys: overall, girls had greater odds of high internalising scores, and female sex, independent of activity and fitness levels, was associated with high emotional difficulties in one mode of covariation between active lifestyle and mental health.

\section{Strengths and limitations}

A key strength is the use of complementary univariate and multivariate analysis approaches to explore links between activity and mental health. Analyses revealed similar patterns of associations: in particular, activity and fitness are more strongly associated with internalising (peer and emotional) problems than with externalising (conduct and hyperactivity) symptoms. We accounted for potential sample bias by imputing missing data: secondary analyses showed favourable relationships between fitness, physical activity and mental health were robust.

Limitations were that we did not collect additional information about the intensity, duration or context of physical activity behaviour, or about sedentary behaviour. Although the multistage fitness test is a reliable and valid measure, ${ }^{47} \mathrm{PE}$ lessons can be a sub-optimal testing environment. We used single-item self-report measures of physical activity and, although we adjusted regression models for a range of confounding factors, we acknowledge more may be present. Considering the context of activity-and inactivity-could deepen our understanding of how it relates to mental health: for example, team sport has been linked to good mental health ${ }^{48}$ while screen-based sedentary behaviour is associated with depressive symptoms. ${ }^{49}$ Cronbach's alpha for the peer, conduct and pro-social subscales of the SDQ was $<0.7$, in line with evidence that, in community samples, these more focused scales might not reliably capture distinct aspects of mental health. ${ }^{35}$ 


\section{Recommendations for future studies}

Our design does not support conclusions about causality: the Fit to Study cluster-randomised controlled trial ${ }^{26}$ aims to establish causation. Future studies could explore the role of muscular fitness, and measure relationships between mental health and physical activity in different contexts, including leisure time and competition.

\section{CONCLUSION}

Fitness as well as physical activity is associated with fewer adolescent psychosocial problems. Fitness assessments could complement the physical activity measures that are essential for public health monitoring. A physically active lifestyle is linked with better overall mental health during early adolescence, a period of rapid developmental change when disorders are becoming established. Further evidence of effect direction is needed.

\section{Author affiliations}

${ }^{1}$ Wellcome Centre for Integrative Neuroimaging, Nuffield Department of Clinical Neurosciences, University of Oxford, Oxford, UK

${ }^{2}$ Centre for Movement, Occupational and Rehabilitation Sciences, Oxford Institute of Nursing, Midwifery and Allied Health Research, Oxford Brookes University, Oxford, UK

${ }^{3}$ Nuffield Department of Population Health, Big Data Institute, University of Oxford, Oxford, UK

Acknowledgements We thank the Fit to Study Investigators (URL: https://www.fitto-study.org/investigators/), for helping plan and deliver the Fit to Study trial and particularly Emma Eldridge, Emily Curtis, Oliver Bushnell and Emily Plester. Thanks to Catharine Creswell, Lucy Bowes, Alan Stein, Kathryn Atherton and Josh de Leeuw for their advice. The authors are grateful to the teachers and pupils at participating schools for their time.

Contributors HJ-B and HD conceived the study. CW, TW and NB acquired the data. TW, PS, TN, HJ-B and CW analysed and interpreted the data. CW drafted the manuscript. All authors critically reviewed the manuscript for important intellectual content and approved the final version for publication.

Funding This work was supported by the Education Endowment Foundation and The Wellcome Trust's Education and Neuroscience Programme [grant number 2681]. HJ-B is funded by the Wellcome Trust (110027/Z/15/Z) and the NIHR Oxford Biomedical Research Centre. The Wellcome Centre for Integrative Neuroimaging is supported by core funding from the Wellcome Trust (203139/Z/16/Z). HD is supported by the Elizabeth Casson Trust and the NIHR Oxford Biomedical Research Centre. The views expressed are those of the authors and not necessarily those of the NHS, the NIHR or the Department of Health.

Competing interests None declared.

Ethics approval Central University Research Ethics Committee of the University of Oxford (R48879).

Provenance and peer review Not commissioned; externally peer reviewed.

Data availability statement The authors agree to share anonymised data upon reasonable request.

Supplemental material This content has been supplied by the author(s). It has not been vetted by BMJ Publishing Group Limited (BMJ) and may not have been peerreviewed. Any opinions or recommendations discussed are solely those of the author(s) and are not endorsed by BMJ. BMJ disclaims all liability and responsibility arising from any reliance placed on the content. Where the content includes any translated material, BMJ does not warrant the accuracy and reliability of the translations (including but not limited to local regulations, clinical guidelines, terminology, drug names and drug dosages), and is not responsible for any error and/or omissions arising from translation and adaptation or otherwise.

Open access This is an open access article distributed in accordance with the Creative Commons Attribution 4.0 Unported (CC BY 4.0) license, which permits others to copy, redistribute, remix, transform and build upon this work for any purpose, provided the original work is properly cited, a link to the licence is given, and indication of whether changes were made. See: https://creativecommons.org/ licenses/by/4.0/.

\section{ORCID iDs}

Catherine Wheatley http://orcid.org/0000-0002-3930-3194

Thomas Wassenaar http://orcid.org/0000-0003-3898-8643

\section{REFERENCES}

1 Guthold R, Stevens GA, Riley LM, et al. Global trends in insufficient physical activity among adolescents: a pooled analysis of 298 population-based surveys with 1.6 million participants. Lancet Child Adolesc Heal 2020;4:23-35.

2 World Health Organisation. Global recommendations on physical activity for health. Geneva: World Health Organization, 2010.

3 Sawyer SM, Afifi RA, Bearinger LH, et al. Adolescence: a foundation for future health. Lancet 2012;379:1630-40.

4 Kessler RC, Berglund P, Demler O, et al. Lifetime prevalence and age-of-onset distributions of DSM-IV disorders in the national comorbidity survey replication. Arch Gen Psychiatry 2005;62:593-602.

5 Rodriguez-Ayllon M, Cadenas-Sánchez C, Estévez-López F, et al. Role of physical activity and sedentary behavior in the mental health of preschoolers, children and adolescents: a systematic review and meta-analysis. Sport Med 2019;1-28.

6 Reiss F. Socioeconomic inequalities and mental health problems in children and adolescents: a systematic review. Soc Sci Med 2013;90:24-31.

7 Biddle S. Physical activity and mental health: evidence is growing. World Psychiatry 2016;15:176.

8 Bailey AP, Hetrick SE, Rosenbaum S, et al. Treating depression with physical activity in adolescents and young adults: a systematic review and meta-analysis of randomised controlled trials. Psychol Med 2018;48:1068-83.

9 Ekeland E, Heian F, Hagen KB. Can exercise improve self esteem in children and young people? A systematic review of randomised controlled trials. Br J Sports Med 2005;39:792-8.

10 Biddle SJH, Ciaccioni S, Thomas G, et al. Physical activity and mental health in children and adolescents: an updated review of reviews and an analysis of causality. Psychol Sport Exerc 2019;42:146-55.

11 Allison KR, Adlaf EM, Irving HM, et al. Relationship of vigorous physical activity to psychologic distress among adolescents. J Adolesc Heal 2005;37:164-6.

12 McMahon EM, Corcoran P, O'Regan G, et al. Physical activity in European adolescents and associations with anxiety, depression and well-being. Eur Child Adolesc Psychiatry 2017;26:111-22.

13 Griffiths L, Geraci M, Cortina-Borja M, et al. Associations between children's behavioural and emotional development and objectively measured physical activity and sedentary time: findings from the UK millennium cohort study. Longit Life Course Stud 2016;7:124-43.

14 Kuiper J, Broer J, van der Wouden JC, van der Wouden JC. Association between physical exercise and psychosocial problems in 96617 Dutch adolescents in secondary education: a cross-sectional study. Eur J Public Health 2018;28:468-73.

15 Ortega FB, Ruiz JR, Castillo MJ, et al. Physical fitness in childhood and adolescence: a powerful marker of health. Int J Obes 2008;32:1.

16 Ruiz JR, Castro-Piñero J, Artero EG, et al. Predictive validity of health-related fitness in youth: a systematic review. Br J Sports Med 2009;43:909-23.

17 Ekelund U, Anderssen SA, Froberg K, et al. Independent associations of physical activity and cardiorespiratory fitness with metabolic risk factors in children: the european youth heart study. Diabetologia 2007;50:1832-40

18 Kandola A, Ashdown-Franks G, Stubbs B, et al. The association between cardiorespiratory fitness and the incidence of common mental health disorders: a systematic review and meta-analysis. J Affect Disord 2019.

19 Tacchi MJ, Heggelund J, Scott J. Predictive validity of objective measures of physical fitness for the new onset of mental disorders in adolescents and young adults. Early Interv Psychiatry 2019;13:1310-18.

20 Lang JJ, Larouche R, Tremblay MS. The association between physical fitness and health in a nationally representative sample of Canadian children and youth aged 6 to 17 years. Heal Promot Chronic Dis Prev Canada Res Policy Pract 2019;39:104-11.

21 Janssen A, Leahy AA, Diallo TMO, et al. Cardiorespiratory fitness, muscular fitness and mental health in older adolescents: a multi-level cross-sectional analysis. Prev Med 2020;132:105985. 
22 Sandercock G, Jones B. Is it time to give population health surveillance a late fitness test? BMJ Publishing Group Ltd and British Association of Sport and Exercise Medicine, 2019.

23 Lang JJ, Tomkinson GR, Janssen I, et al. Making a case for cardiorespiratory fitness surveillance among children and youth. Exerc Sport Sci Rev 2018;46:66-75.

24 Warren JM, Ekelund U, Besson $\mathrm{H}$, et al. Assessment of physical activity: a review of methodologies with reference to epidemiological research: a report of the exercise physiology section of the european association of cardiovascular prevention and rehabilitation. Eur J Cardiovasc Prev Rehabil 2010;17:127-39.

25 DeFina LF, Haskell WL, Willis BL, et al. Physical activity versus cardiorespiratory fitness: two (partly) distinct components of cardiovascular health? Prog Cardiovasc Dis 2015;57:324-9.

26 Wassenaar TM, Wheatley CM, Beale N, et al. Effects of a programme of vigorous physical activity during secondary school physical education on academic performance, fitness, cognition, mental health and the brain of adolescents (fit to study): study protocol for a cluster-randomised trial. Trials 2019;20:189.

27 Leger LA, Mercier D, Gadoury C, et al. The multistage 20 metre shuttle run test for aerobic fitness. J Sports Sci 1988;6:93-101.

28 Mayorga-Vega D, Aguilar-Soto P, Viciana J. Criterion-related validity of the 20-m shuttle run test for estimating cardiorespiratory fitness: a meta-analysis. J Sport Sci Med 2015 August;14:536-47.

29 Tomkinson GR, Lang JJ, Tremblay MS, et al. International normative 20 m shuttle run values from 1142026 children and youth representing 50 countries. Br J Sport Med 2017;51:1545-54.

30 Scott JJ, Morgan PJ, Plotnikoff RC, et al. Reliability and validity of a single-item physical activity measure for adolescents. J Paediatr Child Health 2015;51:787-93.

31 Hagger MS, Chatzisarantis N, Biddle SJH, et al. Antecedents of children's physical activity intentions and behaviour: predictive validity and longitudinal effects. Psychol Heal 2001;16:391-407.

32 Michie S, Johnston M, Francis J, et al. From theory to intervention: mapping theoretically derived behavioural determinants to behaviour change techniques. App/ Psychol 2008;57:660-80.

33 Goodman R. The strengths and difficulties questionnaire: a research note. J Child Psychol Psychiatry 1997;38:581-6.

34 Goodman R, Meltzer H, Bailey V. The strengths and difficulties questionnaire: a pilot study on the validity of the self-report version. Eur Child Adolesc Psychiatry 1998;7:125-30.

35 Goodman A, Lamping DL, Ploubidis GB, et al. When to use broader internalising and externalising subscales instead of the hypothesised five subscales on the strengths and difficulties questionnaire (SDQ): data from british parents, teachers and children. J Abnorm Child Psychol 2010;38:1179-91.

36 Marsh HW, Martin AJ, Jackson S. Introducing a short version of the physical self description questionnaire: new strategies, short-form evaluative criteria, and applications of factor analyses. J Sport Exerc Psychol 2010;32:438-82.

37 Bates D, Sarkar D, Bates MD, et al. The Ime4 package. $R$ Packag Vers 2007; $2: 74$

38 Winkler AM, Webster MA, Vidaurre D, et al. Multi-level block permutation. Neuroimage 2015;123:253-68.

39 Orth U, Robins RW. Understanding the link between low self-esteem and depression. Curr Dir Psychol Sci 2013;22:455-60.

40 Dale LP, Vanderloo L, Moore S, et al. Physical activity and depression, anxiety, and self-esteem in children and youth: an umbrella systematic review. Ment Health Phys Act 2019;16:66-79.

41 Meltzer H, Gatward R, Goodman R, et al. Mental health of children and adolescents in Great Britain. Int Rev Psychiatry 2003;15:185-7.

42 Gerber M, Pühse U. Do exercise and fitness protect against stressinduced health complaints? A review of the literature. Scand J Public Health 2009;37:801-19.

43 Dishman RK, Berthoud H, Booth FW, et al. Neurobiology of exercise. Obesity 2006;14:345-56.

44 Kriemler S, Meyer U, Martin E, et al. Effect of school-based interventions on physical activity and fitness in children and adolescents: a review of reviews and systematic update. $\mathrm{Br} J$ Sports Med 2011;45:923-30

45 Gunnell D, Kidger J, Elvidge H. Adolescent mental health in crisis. BMJ 2018:k2608.

46 Brodersen NH, Steptoe A, Williamson S, et al. Sociodemographic, developmental, environmental, and psychological correlates of physical activity and sedentary behavior at age 11 to 12 . Ann Behav Med 2005;29:2-11.

47 Ruiz JR, Castro-Piñero J, España-Romero V, et al. Field-based fitness assessment in young people: the ALPHA health-related fitness test battery for children and adolescents. $\mathrm{Br} J$ Sports Med 2011;45:518-24.

48 Doré I, O'Loughlin JL, Beauchamp G, et al. Volume and social context of physical activity in association with mental health, anxiety and depression among youth. Prev Med 2016;91:344-50.

49 Hoare E, Milton K, Foster C, et al. The associations between sedentary behaviour and mental health among adolescents: a systematic review. Int J Behav Nutr Phys Act 2016;13:108. 